\title{
FIRST SYNTHESIS, ROTAMERISM AND HERBICIDAL EVALUATION OF SUBSTITUTED $s$-TRIAZINES WITH AMINO-1,3-DIOXANE GROUPS
}

\author{
Marijana Fazekas," Mircea Darabantu, a,b Monica Pintea," Pedro Lameiras, \\ Constantin Bele, ${ }^{c}$ Camelia Berghian ${ }^{a b}$ and Nelly Ple $e^{b}$ \\ “"Babes-Bolyai" University, Department of Organic Chemistry, II Aranyi Jànos str., RO-400028 Cluj-Napoca, Romania \\ Université de Rouen, Institut de Recherche en Chimie Organique Fine (I. R C. O. F.), BP-08, F- 76131 Mont Saint-Aignan Codex, France \\ 'University of Agricultural Sciences and Veterinary Medicine, 3-5 Manastur str., RO-400375 Cluj-Napoca, Romania \\ E-mail : darab@chem.ubbcluj.ro; darabantu@cluj.astral.ro; FAX: 00402645908 18; Tel.: 0040264593833
}

\begin{abstract}
First pure enantiomeric 5-amino-1,3-dioxane, obtained by total diastereospecific ring closure of (1S,2S)-2-amino-1-(4-ni-trophenyl)-1,3-propanediol ("nitrophenylserinol") reacted with cyanuryl chloride to afford $N$-substituted amino-s-triazines and melamine. Their rotameric behaviour around the $\mathrm{C}^{\mathrm{sp} 2}$ (s-triazine)-N(1,3-dioxane) bond is discussed in terms of NMR, as steric and electronic influence of the substituents. The herbicidal evaluation of one of the new compounds is also described.
\end{abstract}

\section{Introduction}

We have previously reported our methodology to prepare pure enantiomeric 5-amino-1,3-dioxanes ( 1 5 ) by direct diastereospecific ring closure of (1S,2S)-2-amino-1-(4-nitrophenyl)-1,3-propanediol (the so called "nitrophenylserinol") and its $N, N$-dimethyl analogue upon treatment with certain aldehydes in strong acidic media $\left(98 \% \mathrm{H}_{2} \mathrm{SO}_{4}, 0^{\circ} \mathrm{C}\right)$. These aminodioxanes exhibited useful reactivity upon treatment with typical electrophiles: aryl(di)aldehydes and acid (poly)chlorides (1-3).

For the present preliminary communication, our outstanding attention is dedicated to the reaction between one representative compound in this class and cyanuryl chloride with a concise stereochemical approach of the products. To the best of our knowledge, amino-1,3-dioxanes were never considered as nucleophiles against cyanuryl chloride though some $N$-subsituted-amino-striazines bearing an acetal motif are mentioned in the literature to be potential anticancer agents (6).

\section{Results and Discussions}

\section{Synthesis}

The starting amino-1,3-dioxane (hereafter abbreviated as $\mathrm{DX}-\mathrm{NH}_{2}$ ) was prepared from the enantiomerically pure (1S,2S)-2-amino-1-(4nitrophenyl)-1.3-propanediol (ni- trophenylserinol) (Scheme-1). The synthesis and stereochemistry of $\mathrm{DX}-\mathrm{NH}_{2}$ we reported elsewhere $(1,4)$. The compound is a stable crystalline solid and an ananchomeric structure, possessing the aromatic group in equatorial position. It is already useful to observe that in $\mathrm{DX}-\mathrm{NH}_{2}$ the amino group is placed in

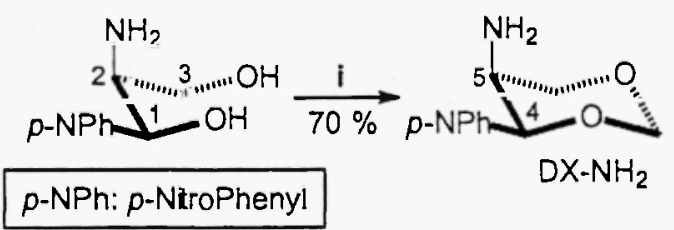

i: 1 eq. $\mathrm{CH}_{2} \mathrm{O} / 10$ eq. $\mathrm{H}_{2} \mathrm{SO}_{4} 98 \% / 24$ hrs. from $0^{\circ} \mathrm{C}$ to r.t.

Scheme-1 axial position flanked by the preferred bisectional orientation of the aromatic ring (cis relationship).

The nucleophilicity of DX-NH $\mathrm{N}_{2}$ against chloro-s-triazines was straightforward (Scheme 2, the partial conversions of cyanuryl chloride are presented in round brackets).

In a first effort (route i) we obtained the expected 1 with a satisfactory yield; TLC monitoring of the reaction evidenced also the noticeable presence of the unreacted starting materials. Attempting at a melamine based exclusively on $\mathrm{DX}-\mathrm{NH}_{2}$ (route ii) failed: 

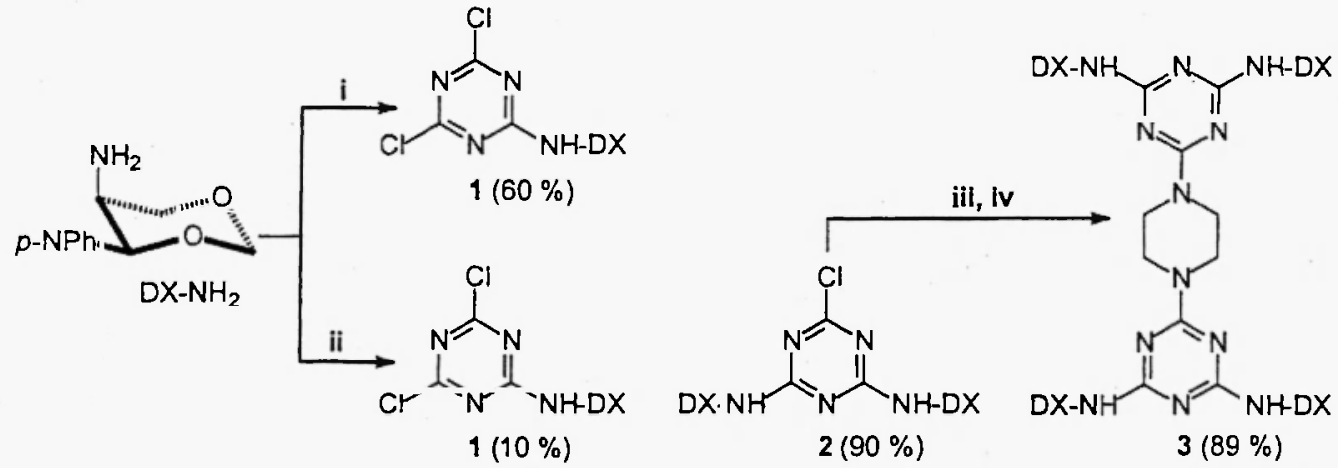

i: 1.05 eq. $\mathrm{C}_{3} \mathrm{~N}_{3} \mathrm{Cl}_{3} / 1.00$ eq. anh. $\mathrm{K}_{2} \mathrm{CO}_{3} / \mathrm{THF} / 24$ hrs. from $0{ }^{\circ} \mathrm{C}$ to r.t.; ii: 0.33 eq. $\mathrm{C}_{3} \mathrm{~N}_{3} \mathrm{Cl}_{3} / 1.00$ eq. anh. $\mathrm{K}_{2} \mathrm{CO}_{3} /$ toluene / 24 hrs. at reflux; iii: 0.49 eq. piperazine hexahydrate, $1.15 \mathrm{eq} . \mathrm{HCl} / i$ $\mathrm{PrOH}$; iv: 0.49 eq. piperazine hydrochloride / 2.00 eq. anh. $\mathrm{K}_{2} \mathrm{CO}_{3} /$ toluene $/ 12 \mathrm{hrs}$. at reflux.

\section{Scheme-2}

the successive replacement of chlorine in cyanuryl chloride stopped after the second substitution. Instead, the chloro-diamino-s-triazine 2 was isolated with excellent yield, together with 1 as side product. They were separated by flash column chromatography. Matching results we reached when the proton scavenger was the "proton sponge" (1,8-bis-dimethylaminonaphthalene). However, if reducing the amount of cyanuryl chloride ( 0.47 eq., 1.00 eq. $\mathrm{K}_{2} \mathrm{CO}_{3}$ ), the partial conversions changed as $47 \% 1$ and $53 \% 2$. Thus, it was impossible to link three DX-NH units to the triazine ring, presumably because the intimate stereochemistry of the DX-NH which influenced the nucleophilicity of the 5-amino group. Consequently, in order to access melamines based on aminodioxane $\mathrm{DX}-\mathrm{NH}_{2}$, our option focused on a stronger nucleophile, piperazine (Scheme-2, routes iii, iv). Its hygroscopicity was avoided by preliminary conversion into hydrochloride. The "dimeric" melamine 3 was prepared in good yield in a very clean reaction.

\section{Stereochemistry and Rotameric Behaviour}

The compound 1 was an ananchomeric structure exhibiting a double bond character of the linkage C-6(s-triazine)-N(1,3-dioxane), hence a restricted rotation in this sequence (Scheme-3). Thus, in the ${ }^{13} \mathrm{C}$ NMR spectrum, the triazinic positions 2 and 4 were found diastereotopic, $\Delta \delta=0.5 \mathrm{ppm}$. In the ${ }^{1} \mathrm{H}$ NMR spectrum, the protons $\mathrm{N} H$ displayed a typical splitting ${ }^{3} J=9.4 \mathrm{~Hz}$ to support a fixed location and an anti arrangement against the proton $\mathrm{H}-5$. Next, one can also anticipate some hindered rotation about the axial $\mathrm{C}-5-\mathrm{N}$ bond, due to the proximity of the ligands $\mathrm{H}-6-$ eq. and $p$ - $\mathrm{NPh}$, predicting an out orientation of the triazinic moiety.

In the case of compound 2, the NMR analysis revealed the diastereomerism issued from the restricted rotation about the

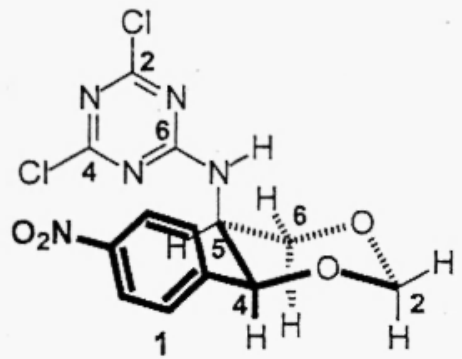

Scheme-3 $\mathrm{C}^{\mathrm{sp2}}$ (triazine)-N(dioxane) bond as mixtures of three blocked rotamers $(8,9)$ designed as: syn-syn $(s-s)$, syn-anti $(s-a)$ and anti-anti $(a-a)$. The dioxane fragments and the triazine chlorine were chosen as references for these descriptors (Scheme-4, Table-1, Figure-1; in Scheme-4 the $p$-nitro group was omitted for reason of simplicity).

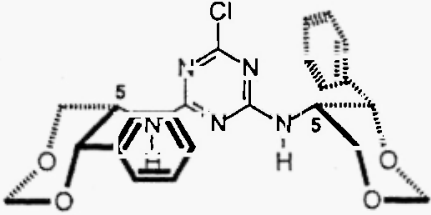

$2(s-s)$

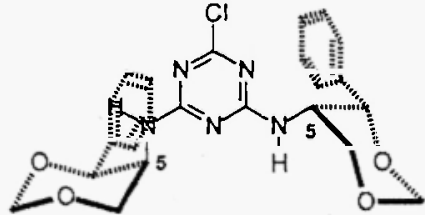

$2(a-s)$

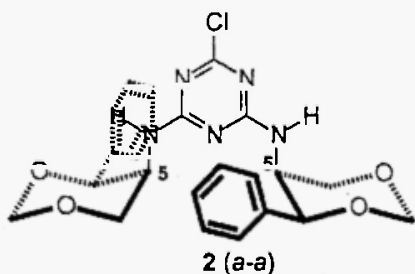

Scheme-4 
Table-1: Relevant ${ }^{1} \mathrm{H}$ NMR data and contributions of the blocked rotamers for the compound 2

\begin{tabular}{ccccccc}
\hline Solvent & \multicolumn{3}{c}{$\begin{array}{c}\text { Rotamers (\%) } \\
\text { according } \\
\text { to NH signals }\end{array}$} & \multicolumn{3}{c}{$\begin{array}{c}\delta_{\mathrm{NH}}(\mathrm{ppm}) \\
\left({ }^{3} \mathrm{JNH}-\boldsymbol{H}-\mathbf{H z}\right)\end{array}$} \\
\cline { 2 - 8 } & $(s-s)$ & $(s-a)$ & $(a-a)$ & $(s-s)$ & $(s-a)$ & $(a-a)$ \\
\hline DMSO-d $d_{6}$ & 34 & 53 & 13 & $7.55(8.8)$ & $\begin{array}{c}7.55,7.13 \\
(8.8),(9.6)\end{array}$ & $\begin{array}{c}7.10 \\
\end{array}$ \\
& & & & & $(11.6)$ \\
\hline $\mathrm{C}_{6} \mathrm{D}_{6}$ & 53 & 24 & 23 & $6.48(8.3)$ & $5.57,5.78$ & 5.85 \\
& & & & & $(9.1),(9.8)$ & $(9.8)$ \\
\hline $\mathrm{CDCl}_{3}$ & 26 & 54 & 20 & $6.09(9.4)$ & $5.88,5.71$ & 6.00 \\
& & & & & $(9.4),(9.8)$ & $(9.8)$ \\
\hline
\end{tabular}

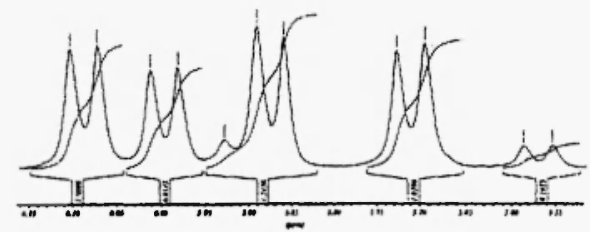

'H NMR spectrum of the compound 2 (300 $\mathrm{MHz}, \mathrm{CDCl}_{3}, 293 \mathrm{~K}$ ), detail in the region of the protons $\mathrm{N} H$.

Figure-1

As expected, the "reference" protons were $\mathrm{N} H$ and used for the calculations (Table-1): isochronous doublets in environments $(s-s)$ and $(a-a)$ but anisochronous in $(s-a)$. The rotamerism appeared influenced by the stereochemistry of the axial linkage dioxane-triazine, (Figure-1). Surprisingly, the NMR spectra of 2 clearly indicated that this molecule, arising from a bulky nucleophile, can adopt all possible spatial arrangements $(s-s, s-$ $a, a-a)$ suggested by the manipulation of the Drieding models (Scheme-4, Figure -1). Moreover, as shown in Figure-1, in $\mathrm{CDCl}_{3}$, another pair of doublets was revealed to indicate a fourth minor rotamer which was not assigned. It must be observed that, in all stereoisomers, the magnitude of the coupling pattern ${ }^{3} J$ between protons $\mathrm{NH}$ and $\mathrm{H}$-5-eq. was significant, in agreement with some hindrance to rotation about the axial $\mathrm{C}-5-\mathrm{NH}$ bond (Table-1). A major dependence on the solvent was determined related to the content of rotamers: the statistically favoured $(s-a)$ rotamer was dominant in polar and chelating solvent (DMSO- $d_{6}$ ) or only polar $\left(\mathrm{CDCl}_{3}\right)$. In contrast, the A.S.I.S. (10) interactions required the rotamer $(s-s)$ as prevailing.

The NMR experiments carried out in DMSO- $d_{6}$ by increasing the temperature $(293 \rightarrow 353 \mathrm{~K})$ provided the results depicted in Figure-2: one can not assume that even at $80{ }^{\circ} \mathrm{C}$ the compound 2 reached complete flexibility.

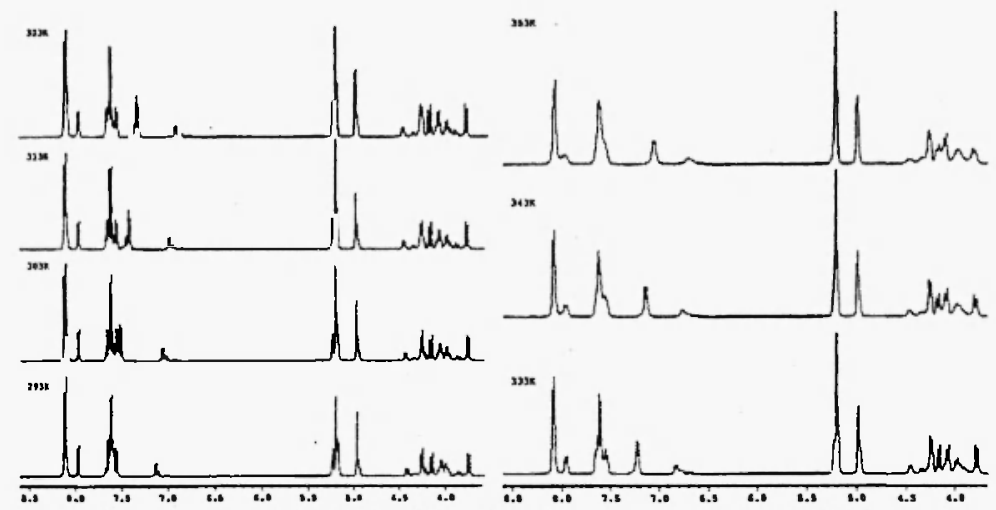

'H DNMR spectrum of the compound $2(400 \mathrm{MHz}, 353 \mathrm{~K}$, see also Scheme-3) from downfield to upfield $\delta$ (ppm), $J(\mathrm{~Hz}): 8.08$ (d, $\left.{ }^{3} J=8.0 \mathrm{~Hz}, \mathrm{H}-p-\mathrm{NPh}\right), 7.97(\mathrm{bs}, p-\mathrm{NPh}), 7.62\left(\mathrm{~d},{ }^{3} J=6.8 \mathrm{~Hz}, p-\mathrm{NPh}\right), 7.06\left(\mathrm{~d},{ }^{3} J=8.4 \mathrm{~Hz}\right.$, NH), 6.69 (bs, NH), 5.21 (s, H-4-ax.), 5.20 (d, ${ }^{2} J=6.0 \mathrm{~Hz}, \mathrm{H}-2$-eq.), 4.99 (d, ${ }^{2} J=6.0, \mathrm{H}-2$-ax.), 4.46 (bs, H-5-eq.), 4.35 (d, ${ }^{2} J=7.6 \mathrm{~Hz}, \mathrm{H}-5$-eq.), 4.26 (d, ${ }^{3} J=8.4 \mathrm{~Hz}, \mathrm{H}-5$-eq.), 4.17 (d, ${ }^{2} J=10.8 \mathrm{~Hz}, \mathrm{H}-6-\mathrm{eq}$ ), 4.09 (d, ${ }^{2} J=11.2 \mathrm{~Hz}, \mathrm{H}-$ 6-eq.), 3.97 (bs, H-6-ax.), 3.80 (d, ${ }^{2} J=10.8 \mathrm{~Hz}, \mathrm{H}-6-\mathrm{ax}$. ).

Figure-2

Indeed, at $353 \mathrm{~K}$, three correlations between $\mathrm{NH}-\mathrm{H}$-5-eq. were assigned by COSY Experiments, consistent with three unequal environments for the proton $\mathrm{H}-5$-eq.: two doublets and one broad singlet.

The "dimeric" melamine 3 can exist as seven distinct rotamers (Scheme-5, the 1,3-dioxane and piperazine rings as references; the syn and anti descriptors are cited clockwise) (8). At room temperature, the ${ }^{1} \mathrm{H}$ NMR $300 \mathrm{MHz}$ spectra $\left(\mathrm{CDCl}_{3}\right.$ and DMSO- $\left.d_{6}\right)$ were complex and allowed only to identify the type of compound as the envisaged one. The ${ }^{1} \mathrm{H}$ DNMR (400 MHz, DMSO- $d_{6}$ ) recorded by rising the temperature $(\Delta T=10 \mathrm{~K})$ provided at $80^{\circ} \mathrm{C}$ a single deblocked structure in a slow exchange domain between sites (Figure-3). 


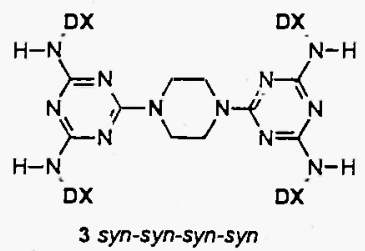<smiles>[R]OC(=O)Nc1nc(N[R]([R])=O)nc(N2CCN(c3nc(NC(=O)Nc4ccccc4)nc(NC(=O)c4ccccc4)n3)CC2)n1</smiles>

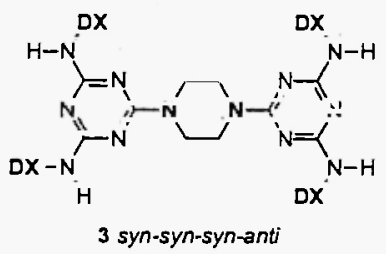

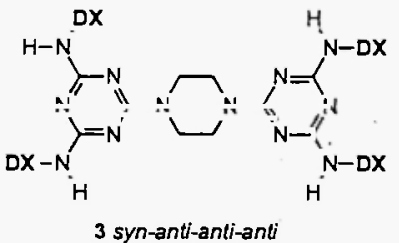<smiles>[R4]NC1=NC(N[R]([H])=O)NC(N2CCN(c3nc(N[R4])nc(N[R2])n3)CC2)=N1</smiles>

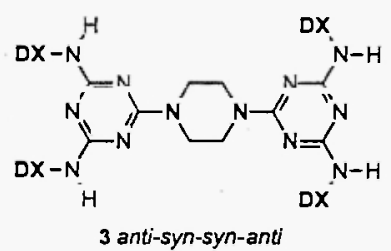<smiles>[R20]Nc1nc(N[R20])nc(N2CCN(c3nc(N[R20])nc(N[R])n3)CC2)n1</smiles>
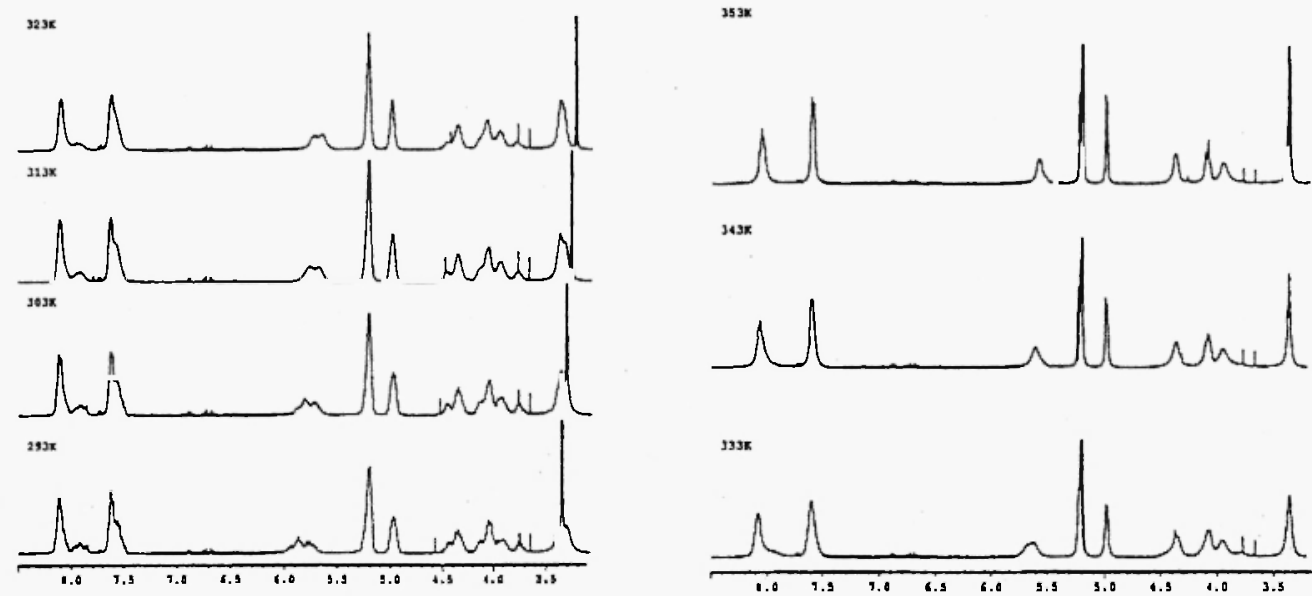

't1 DNMR spectrum of the compound $3(400 \mathrm{MHz}, 353 \mathrm{~K}$, see also Scheme-5) from downfield to upfield $\delta$ ( $\mathrm{ppm}), J(\mathrm{~Hz}): 8.06(8 \mathrm{H}, \mathrm{bs}, \mathrm{H}-p-\mathrm{NPh}), 7.59\left(8 \mathrm{H}, \mathrm{d},{ }^{3} J=7.6 \mathrm{~Hz}, \mathrm{H}-p-\mathrm{NPh}\right), 5.58\left(4 \mathrm{H}, \mathrm{d},{ }^{3} J=7.6 \mathrm{~Hz}, \mathrm{NH}\right), 5.22$ $\left(4 \mathrm{H}, \mathrm{d},{ }^{2} \mathrm{~J}=6.0 \mathrm{~Hz}, \mathrm{H}-2\right.$-eq.), $5.20\left(4 \mathrm{H}, \mathrm{s}, \mathrm{H}-4\right.$-ax.), $4.99\left(4 \mathrm{H}, \mathrm{d},{ }^{2} J=6.0 \mathrm{~Hz}, \mathrm{H}-2\right.$-ax.), $4.37\left(4 \mathrm{H}, \mathrm{d},{ }^{3} \mathrm{~J}=7.6 \mathrm{~Hz}\right.$, H-5-eq.), 4.10 (4 H, d, ${ }^{2} J=10.4 \mathrm{~Hz}, \mathrm{H}$-6-eq.), 3.97 ( $4 \mathrm{H}$, bs, $\mathrm{H}-6$-ax.), $3.36\left(8 \mathrm{H}, \mathrm{s}, \mathrm{CH}_{2}\right.$, piperazine).

\section{Herbicidal evaluation}

Figure-3

Compound $\mathbf{2}$ was in addition tested as potential herbicide on seeds of Cucumis sativus and Raphanus sativus. Literature methods were straightforward (11). Atrazine ${ }^{\oplus}$ was used for testing biological activity along with the synthesised compound 2 . The results, as mean $( \pm S D)$ percentage values of germination inhibition and root length are collected in Table-2. 
Table-2: Percent inhibitions of seeds germination and root length of Cucumis sativus and Raphanus sativus in response to different concentrations of the compound 2 as compared to those of Atrazine ${ }^{\otimes}$

\begin{tabular}{lccccc}
\hline Tested species & & \multicolumn{2}{c}{ Germination } & \multicolumn{2}{c}{ Root length $^{-}$} \\
\cline { 2 - 6 } & Conc. & $\mathbf{2}$ & Atrazine $^{\otimes}$ & $\mathbf{2}$ & Atrazine $^{\otimes}$ \\
\hline Cucumis & $0.50 \mathrm{mM}$ & $57 \pm 4.9$ & $62 \pm 4.2$ & $67 \pm 5.6$ & $69 \pm 6.5$ \\
sativus & $0.75 \mathrm{mM}$ & $84 \pm 2.3$ & $89 \pm 2.5$ & $86 \pm 3.4$ & $91 \pm 3.3$ \\
& $1.00 \mathrm{mM}$ & $100 \pm 0.0$ & $100 \pm 0.0$ & - & - \\
\hline Raphanus & $0.50 \mathrm{mM}$ & $65 \pm 6.2$ & $71 \pm 5.6$ & $72 \pm 4.8$ & $74 \pm 6.2$ \\
Sativus & $0.75 \mathrm{mM}$ & $87 \pm 3.7$ & $90 \pm 2.4$ & $91 \pm 4.6$ & $93 \pm 5.8$ \\
& $1.00 \mathrm{mM}$ & $100 \pm 0.0$ & $100 \pm 0.0$ & - & - \\
\hline
\end{tabular}

Our opening data evidenced an important inhibition in germination seeds of the tested species, even complete (c. $1 \mathrm{mM}$ ), quite similar to Atrazine ${ }^{\otimes}$. The root length was also significantly reduced.

\section{Experimental \\ General}

Melting points were uncorrected; they were carried out on ELECTROTHERMAL ${ }^{\circledR}$ instrument. Current NMR spectra were recorded on Brucker ${ }^{\circledR}$ AM 300 instrument operating at 300 and $75 \mathrm{MHz}$ for ${ }^{1} \mathrm{H}$ and ${ }^{13} \mathrm{C}$ nuclei respectively. The ${ }^{1} \mathrm{H}$ DNMR spectra were run on Brucker ${ }^{\otimes} \mathrm{AM} 400$ instrument operating at $400 \mathrm{MHz}$ for ${ }^{1} \mathrm{H}$ nuclei with each step $10 \mathrm{~K}$ increasing the temperature. No $\mathrm{SiMe}_{4}$ was added; chemical shifts were measured against the solvent peak. All chemical shifts ( $\delta$ values) are given throughout in ppm; all coupling patterns ( ${ }^{\mathrm{n}} \mathrm{J}_{\mathrm{H}, \mathrm{H}}$ values) are given throughout in $\mathrm{Hz}$. TLC was performed by using aluminium sheets with silica gel $60 \mathrm{~F}_{254}\left(\mathrm{Merck}^{\circledR}\right)$; flash column chromatography was conducted on Silica gel Si $60\left(40-63 \mu \mathrm{m}\right.$, Merck $\left.{ }^{3}\right)$. IR spectra were performed on a PerkinElmer ${ }^{\oplus} 16$ PC FT-IR spectrometer. Only relevant absorptions are listed in $\mathrm{cm}^{-1}$ [weak (w), medium $(\mathrm{m})$ or (s) strong]. Mass spectrum (MS) was recorded on an ATI-Unicam Automass ${ }^{\circledR}$ apparatus, fitted (or not) with a GC-mass coupling (high-resolution J\&W column, $30 \mathrm{~m}, 0.25 \mathrm{~mm} \mathrm{ID}$, flow rate: 1.2 $\left.\mathrm{mL} \min ^{-1}\right)$.

2,4-Dichloro-6-[(4S,5S)-4-(4-nitrophenyl)-1,3-dioxan-5-yl]-amino-s-triazine (1): (60\%) yellowish crystalline powder; m.p. $=194-195{ }^{\circ} \mathrm{C}\left(\mathrm{Et}_{2} \mathrm{O}\right)$; [Found: $\mathrm{C}, 42.11 ; \mathrm{H}, 2.77 ; \mathrm{N}, 19.09 . \mathrm{C}_{13} \mathrm{H}_{11} \mathrm{~N}_{5} \mathrm{Cl}_{2} \mathrm{O}_{4}$ requires $\mathrm{C}, 41.96 ; \mathrm{H}, 2.98 ; \mathrm{N}, 18.82 \%$ ]; IR ( $\left.v_{\max }, \mathrm{KBr}\right) 3305(\mathrm{~s}), 2875(\mathrm{~m}), 1585(\mathrm{~s}), 1556(\mathrm{~s}), 1510$ (s), $1410(\mathrm{~s}), 1346(\mathrm{~s}), 1325(\mathrm{~s}), 1240(\mathrm{~m}), 1183(\mathrm{~s}), 1167(\mathrm{~s}), 1103(\mathrm{~s}), 1043(\mathrm{~m}), 1028(\mathrm{~m}), 964(\mathrm{~m})$, $842(\mathrm{~m}), 798(\mathrm{~m}), 713(\mathrm{~m}) \mathrm{cm}^{-1},{ }^{\prime} \square \quad$ पQR $\left(300 \mathrm{MHz}, \mathrm{CDCl}_{3}, 293 \mathrm{~K}\right): 8.18\left(2 \mathrm{H}, \mathrm{d},{ }^{3} \mathrm{~J}=8.7 \mathrm{~Hz}, \mathrm{H}-p-\right.$ $\mathrm{NPh}), 7.51\left(2 \mathrm{H}, \mathrm{d},{ }^{3} J=8.7 \mathrm{~Hz}, \mathrm{H}-p-\mathrm{NPh}\right), 6.65\left(1 \mathrm{H}, \mathrm{d},{ }^{3} J=9.4 \mathrm{~Hz}, \mathrm{NH}\right), 5.35\left(1 \mathrm{H}, \mathrm{d},{ }^{2} J=6.4 \mathrm{~Hz}, \mathrm{H}-\right.$ 2eq.), 5.11 ( $1 \mathrm{H}, \mathrm{s}, \mathrm{H}-4$-ax.), 5.02 (1 H, d, ${ }^{2} J=6.4 \mathrm{~Hz}, \mathrm{H}-2$-ax.), $4.56\left(1 \mathrm{H}, \mathrm{d},{ }^{3} J=9.8 \mathrm{~Hz}, \mathrm{H}-5\right.$-eq. $), 4.24$ (1 H, d, ${ }^{2} J=12.1 \mathrm{~Hz}, \mathrm{H}-6$-eq. $), 4.14\left(1 \mathrm{H}, \mathrm{d},{ }^{2} J=11.3 \mathrm{~Hz}, \mathrm{H}-6\right.$-ax. $) ;{ }^{13} \mathrm{C} \mathrm{NMR}\left(75 \mathrm{MHz}, \mathrm{CDCl}_{3}, 293 \mathrm{~K}\right)$ : $171.1(1 \mathrm{C}, \mathrm{C}-\mathrm{Cl}$ ), $170.6(1 \mathrm{C}, \mathrm{C}-\mathrm{Cl}), 165.8(1 \mathrm{C}, \mathrm{C}-\mathrm{N}), 148.0$ (1 C, Cq. $p$-NPh), 144.4 (1 C, Cq.- $\mathrm{NPh}), 126.8$ (2 C, CH- $p$-NPh), 124.0 (2 C, CH- p-NPh), 94.9 (1 C, C-2), 78.9 (1 C, C-4), 70.6 (1 C, C-6), 50.2 (1 C, C-5); MS (EI, $70 \mathrm{eV}$ ); m/z (rel. int. \%): 371 (40) [M+-1], 341 (25), 311 (100), 277 (18), $218(39), 190(25), 164$ (27).

2-Chloro-4,6-bis[(4S,5S)-4-(4-nitrophenyl)-1,3-dioxan-5-yl]-amino-s-triazine (2): (90\%) yellow crystalline powder; m.p. $=154-155^{\circ} \mathrm{C}$ (flash column chromatography, eluent ligroine : acetone 1.5:1 v/v); [Found: $\mathrm{C}, 48.97 ; \mathrm{H}, 4.14 ; \mathrm{N}, 17.99 . \mathrm{C}_{23} \mathrm{H}_{22} \mathrm{~N}_{7} \mathrm{ClO}_{8}$ requires $\mathrm{C}, 49.34 ; \mathrm{H}, 3.96 ; \mathrm{N}, 17.51 \%$ ]; IR $\left(v_{\max }, \mathrm{KBr}\right) 3404(\mathrm{~m}), 3314(\mathrm{~m}), 2859(\mathrm{~s}), 1573(\mathrm{~s}), 1518(\mathrm{~s}), 1510(\mathrm{~s}), 1346(\mathrm{~s}), 1240(\mathrm{~m}), 1174(\mathrm{~s})$, $1167(\mathrm{~s}), 1094(\mathrm{~s}), 1026(\mathrm{~s}), 1028(\mathrm{~s}), 987(\mathrm{~s}), 851(\mathrm{~m}), 805(\mathrm{~m}), 711(\mathrm{~m}) \mathrm{cm}^{-1} ;{ }^{1} \square \quad \square \square R(300 \mathrm{MHz}$, $\left.\mathrm{CDCl}_{3}, 293 \mathrm{~K}\right): 8.12-8.05(12 \mathrm{H}, \mathrm{m}, \mathrm{H}-p-\mathrm{NPh}), 7.45-7.39(12 \mathrm{H}, \mathrm{m}, \mathrm{H}-p-\mathrm{NPh}), 6.09\left(2 \mathrm{H}, \mathrm{d},{ }^{3} \mathrm{~J}=9.4\right.$ $\left.\mathrm{Hz}, \mathrm{N} H_{s-s}\right), 6.00\left(2 \mathrm{H}, \mathrm{d},{ }^{3} J=9.8 \mathrm{~Hz}, \mathrm{~N} H_{a-a}\right), 5.88\left(1 \mathrm{H}, \mathrm{d},{ }^{3} \mathrm{~J}=9.4 \mathrm{~Hz}, \mathrm{~N} H_{s-a}\right), 5.71\left(1 \mathrm{H}, \mathrm{d},{ }^{3} J=9.8 \mathrm{~Hz}\right.$, $\left.\mathrm{N} H_{s-a}\right)$, 5.35-5.30 (3 H, m, H-2eq.), 5.25-5.20 (3 H, m, H-2eq.), 4.99-4.91 (12 H, m, H-2-ax., H-4-ax.), 4.41-3.88 (18 H, m, H-5-eq., H-6-eq., H-6-ax.); ${ }^{13} \mathrm{C} \mathrm{NMR} \mathrm{(75} \mathrm{MHz,} \mathrm{CDCl}_{3}, 293 \mathrm{~K}$ ): 169.5 (3 C, C- 
Cl), 165.8, 165.4, 165.3, 165.2 (6 C, C-N), 147.9, 147.7 (6 C, C-q.- $p$-NPh), 145.2, 145.0 (6 C, Cq.- $\mathrm{NPh}), 126.9,126.73,126.66$ (12 C, CH- $p$-NPh), 123.80, 123.77, 123.73 (12 C, CH- $p$-NPh), 94.9, y4.8 (6 C, C-2), 79.33, 79.27, 79.21, 79.1 (6 C, C-4), 71.1, 70.8, 70.7, 70.5 (6 C, C-6), 49.6, 49.5,

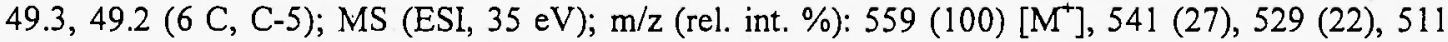
(10).

1,4-Bis $\{4,6$-bis $[(4 S, 5 S)$-4-(4-nitrophenyl)-1,3-dioxan-5-yl]-amino-s-triazin-2-yl\}-piperazine (3): $(89 \%)$ yellow crystalline powder; m.p. $=224-225^{\circ} \mathrm{C}$ (flash column chromatography, eluent ligroine : acetone $1.25: 1 \mathrm{v} / \mathrm{v}$ ); [Found: $\mathrm{C}, 53.37 ; \mathrm{H}, 5.02 ; \mathrm{N}, 19.69 . \mathrm{C}_{50} \mathrm{H}_{52} \mathrm{~N}_{16} \mathrm{O}_{16}$ requires $\mathrm{C}, 53.00 ; \mathrm{H}, 4.63 ; \mathrm{N}$, $19.78 \%$ ]; IR ( $\left.v_{\max }, \mathrm{KBr}\right) 3414(\mathrm{~m}), 2855(\mathrm{~m}), 1576(\mathrm{~s}), 1548(\mathrm{~s}), 1520(\mathrm{~s}), 1442(\mathrm{~s}), 1346(\mathrm{~s}), 1244$ (w), $1173(\mathrm{~s}), 1095(\mathrm{~m}), 1027(\mathrm{~m}), 985(\mathrm{~m}), 852(\mathrm{w}), 810(\mathrm{~m}), 742(\mathrm{w}), 711(\mathrm{w}) \mathrm{cm}^{-1} ;{ }^{1} \square \quad \square \square R(400$ MHz, DMSO- $\left.d_{6}, 353 \mathrm{~K}\right): 8.06\left(8 \mathrm{H}\right.$, bs, H-p-NPh), $7.59\left(8 \mathrm{H}, \mathrm{d},{ }^{3} \mathrm{~J}=7.6 \mathrm{~Hz}, \mathrm{H}-p\right.$-NPh), $5.58(4 \mathrm{H}, \mathrm{d}$, $\left.{ }^{3} J=7.6 \mathrm{~Hz}, \mathrm{NH}\right), 5.22\left(4 \mathrm{H}, \mathrm{d},{ }^{2} J=6.0 \mathrm{~Hz}, \mathrm{H}-2 \mathrm{eq}\right), 5.20(4 \mathrm{H}, \mathrm{s}, \mathrm{H}-4-\mathrm{ax}), 4.99\left(4 \mathrm{H}, \mathrm{d},{ }^{2} J=6.0 \mathrm{~Hz}, \mathrm{H}-2-\right.$ ax.), 4.37 ( $4 \mathrm{H}, \mathrm{d},{ }^{3} \mathrm{~J}=7.6 \mathrm{~Hz}, \mathrm{H}-5$-eq.), $4.10\left(4 \mathrm{H}, \mathrm{d},{ }^{2} J=10.4 \mathrm{~Hz}, \mathrm{H}-6\right.$-eq.), 3.94 (4 H, bs, H-6-ax.), $3.36\left(8 \mathrm{H}, \mathrm{s}, \mathrm{CH}_{2}\right.$ piperazine); ${ }^{13} \mathrm{C} \mathrm{NMR}\left(75 \mathrm{MHz}, \mathrm{CDCl}_{3}, 293 \mathrm{~K}\right) ; 165.7(4 \mathrm{C}, \mathrm{C}-\mathrm{N}), 165.5(2 \mathrm{C}, \mathrm{C}-$ $\mathrm{N}), 147.6$ (4 C, Cq.- $p$-NPh), 146.0 (4 C, Cq.- $p-\mathrm{NPh}), 127.0$ (8 C, CH- $p-\mathrm{NPh}), 123.4$ (8 C, CH- NPh), 94.7 (4 C, C-2), 79.8 (4 C, C-4), 71.4 (4 C, C-6), 49.0 (4 C, C-5), 42.8 (4 C, CHz-piperazine); $\mathrm{MS}\left(\mathrm{FAB}^{+}\right) ; \mathrm{m} / \mathrm{z}$ (rel. int. \%): $1132(95)\left[\mathrm{M}^{+}-1\right], 952(20), 663(33), 551$ (33), 459 (100).

\section{Conclusions}

As demonstrated by our first example, a 5-amino-1,3-dioxane built on $p$-nitrophenyserinol skeleton reacts with cyanuryl chloride to yield amino-s-triazines in medium to good yields. However, the substitution of the third chlorine appears not possible due to the axial orientation of the nucleophilic group linked to an anancomeric skeleton. At room temperature, all $\mathrm{N}$-substituted-amino-s-triazines with a 1,3-dioxane group are distinct type of rotamers due to the partial double bond character of the $\mathrm{C}^{\mathrm{sp} 2}$ (s-triazine)-N(1,3-dioxane) site. The content of rotameric species is also dependent on the solvent. The herbicidal activity in this class of $s$-triazines was tested. The full report of our complete results is under consideration for the near future.

\section{References}

1. M. Darabantu; S. Mager; C. Puscas; M. Bogdan; E. Cotora; G. Plé and I. Bratu, Rev. Rom. Chim. 39, 955-965 (1994).

2. M. Darabantu; S. Mager; C. Puscas; M. Bogdan; G. Plé; E. Cotora and D. Kovacs, Rev. Rom.Chim. 40, 453-461 (1995).

3. M. Darabantu; S. Mager; C. Puscas; G. Plé; M. Bogdan and E. Cotora, Rev. Rom. Chim. 40, 907-916 (1995).

4. M. Darabantu; C. Maiereanu; G. Plé; C. Berghian; E. Condamine and Y. Ramondenc, Heterocyclic Commun. 7(6), 593-596 (2001).

5. M. Darabantu; G. Ple; S. Mager; C. Puscas and E. Cotora, Tetrahedron 53, 1909-1922 (1997).

6. B. O. Kraiz and A. L. Remizov, Zh. Org. Khim. 15(16), 1282-1283 (1979).

7. Recent data, for example: G. Boncic-Caricic; Z. D. Tadic and M. Muskatirovic, J. Serb. Chem. Soc. 59(12), 929-933 (1994); N. D. Reddy; A. Elias and A. Vic, J. Chem. Res. Synop. 9, 504-505 (1998).

8. See our preceding communication.

9. a) A. R. Katritzky; I. Ghiviriga; D. C. Oniciu and A. Barkock, J. Chem. Soc. Perkin Trans. 2, 785-792 (1995); b) A. R. Katritzky; I. Ghiviriga; P. G. Steel and D. C. Oniciu, J. Chem. Soc. Perkin Trans. 2, 443-447 (1996); c) I. Ghiviriga and D. Oniciu, Chem. Comm. 22, 2718-2719 (2002).

10. a) P. Laszlo, Bull. Soc. Chim. Fr. 10, 2658-2661 (1964); b) K. Nikki, Magn. Res. Chem. 28, 385-388 (1990).

11. a) K. Grossmann; S. Tresch and Z. Plath, Naturforsch, 56c, 559-569 (2001); b) H. Omokawa and M. Konnai, Pestic. Sci. 35, $83-86$ (1992); c) H. Omokawa; N. Ichizen and T. Takematsu, Agric. Biol. Chem. 51, 2563-2568 (1987).

Received on January 20, 2005 